\title{
USING CONSUMER ELECTRONICS AND APPS IN INDUSTRIAL ENVIRONMENTS - DEVELOPMENT OF A FRAMEWORK FOR DYNAMIC FEATURE DEPLOYMENT AND EXTENSION BY USING APPS ON FIELD DEVICES
}

\author{
MATHIAS SCHMITT \\ German Research Center for Artificial Intelligence, Innovative Factory Systems, \\ Trippstadter Straße 122, 67663 Kaiserslautern, Germany, mathias.schmitt@dfki.de
}

\begin{abstract}
The aim of this paper is to give a preliminary insight regarding the current work in the field of mobile interaction in industrial environments by using established interaction technologies and metaphors from the consumer goods industry. The major objective is the development and implementation of a holistic app-framework, which enables dynamic feature deployment and extension by using mobile apps on industrial field devices. As a result, field device functionalities can be updated and adapted effectively in accordance with well-known appconcepts from consumer electronics to comply with the urgent requirements of more flexible and changeable factory systems of the future. In addition, a much more user-friendly and utilizable interaction with field devices can be realized. Proprietary software solutions and device-stationary user interfaces can be overcome and replaced by uniform, cross-vendor solutions.
\end{abstract}

Key words: field device integration, mobile interaction, consumer electronics, mobile apps

\section{Introduction}

The increasing individualization requirements, combined with the associated technical heterogeneity and complexity of products, require more flexible factory systems able to cope with today's rapidly changing market needs. Consequently, industrial field devices also need to continuously be adapted to changes in the production process. While nowadays proprietary software tools and stationary, device-linked user interfaces are used to interact with field devices, there is a need for holistic and manufacturer-comprehensive solutions to satisfy the demands of flexibility and adaptability.

The transfer of established interaction technologies and metaphors from the field of consumer electronics into the industrial application appears to be a promising approach. Meant herewith are modular software components in the form of "apps", which are provided via standardized communication interfaces in the network (e.g. "App Store") and allow an effective, user-friendly interaction via uniform, mobile user interfaces (e.g. Tablet, Smartphone). However, the direct mapping of established "app-concepts" in the field of consumer electronics into industrial applications is not trivial, due to the fact that current industrial requirements have not yet been taken into account.

Therefore, the aim is to develop and implement a multi-vendor "app-framework", which enables dynamic feature deployment and extension by using apps on industrial field devices. Thus, a much more user-friendly and utilizable interaction between the user and the field device can be realized.

For the realization of the proposed app-framework, established interaction technologies and metaphors from the consumer goods market are used and consequently adapted for the industrial use. Based on this, a software architecture is developed, which is the basis for the implementation of the app-framework. The development and realization of a technology-demonstrator, as an instantiation of the proposed approach, will serve as a proof of concept.

In the following, the app-framework is presented that enables dynamic feature deployment and extension by using apps on industrial field devices. In section 2 of this paper the related work in the area of field device integration and mobile interaction technologies is presented, followed by the objective and the general approach of the app-framework. Afterwards, in section 4, the general app-framework is 
described and some preliminary insights of the on-going work are shown. The paper ends with concluding remarks and an outlook.

\section{State of the Art}

Shortening innovation and product life cycles, together with a growing demand for customized products, lead to growing demands concerning the flexibility and inconstancy of production processes. However, current factory systems are limited in their ability to cope with these requirements, since they are primarily developed and realized as monolithic systems for performing one specific task (Abele, 2011). Reconfigurable, adaptable production processes usually imply a much more frequent adjustment of equipment and processes. Strongly related to this, industrial field devices must additionally be adapted more frequently to the changing circumstances.

By the use of embedded, intelligent information and communication technologies, the technological enabler to address these challenges is already given. The vision of the so called 4th Industrial Revolution describes in this context the autonomous control of factory systems by using these distributed and collaborating cyber physical systems (Lee, 2008; Broy 2010). In the future, this development will also allow the augmentation of intelligent field devices with modular software components, for ensuring a higher degree of reusability without any hardware set-ups or changes. By the use of modular software components running directly on the field devices, a significantly higher flexibility can be achieved.

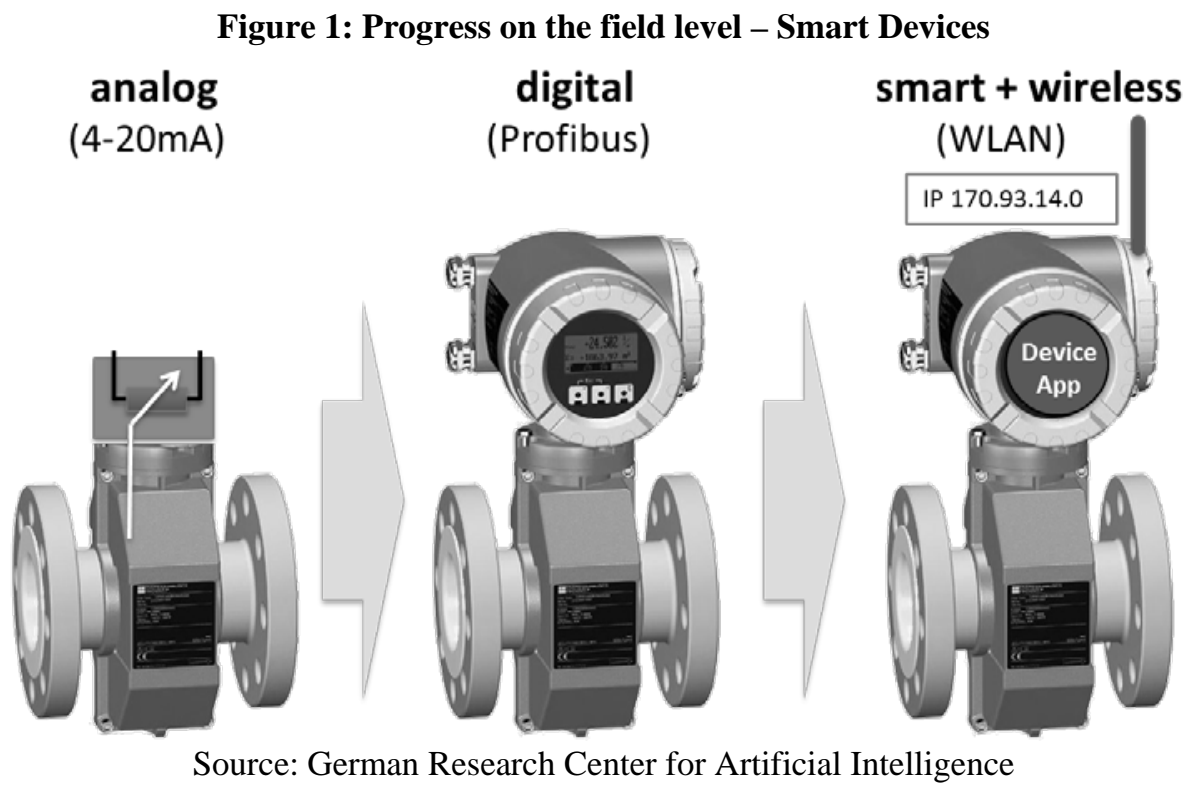

In current factory systems there are basically stationary, proprietary user interfaces for commissioning, monitoring and maintenance of field devices in use. Particular industrial field devices have their own vendor-specific interaction solutions and operating philosophies, that provide the integration of the user interface directly on the field device. With the increasing distribution of these intelligent field devices, the relevance of utilizable user interfaces will increase as well. The human as an integral part of production systems must be supported by smart concepts and methods to reduce complexity and to establish transparency.

With Electronic Device Description Language (EDDL) and Field-Device-Tool/Device-TypeManager (FDT/DTM), two manufacturer-independent approaches have emerged for the parameterization of field devices in industrial automation. By the use of EDD (Electronic Device Description) for the field device integration, device functions and parameters are set by the vendors in a standardized, text based description language (Electronic Device Description Language, short EDDL). Disadvantages of this technology are the limitations in the description of complex, graphical representations and functionalities (EDDL, 2013). The Field Device Tool (FDT) provides a field device integration framework, which allows the management of various device descriptions (Device Type Manager, short DTM). Each DTM is a standalone software object used to interact with the field device and must be installed on the software system which is used. Among compatibility problems, FDT technology disadvantages include the close linking to the DTM provided by the manufacturer to the Windows platform and associated dependencies 
(FDT, 2013). In fact, both approaches do support the commissioning of field devices (e.g. configuration, parameterization). However, they do not address the reconfiguration or the flexible reloading of software functionality into the device. Especially in the context of growing flexibility and adaptability, both EDDL and FDT/DTM are insufficient.

The transfer of established interaction technologies (e.g. Tablets, Smartphones) and metaphors (e.g. multi-touch, apps) for mobile support in the area of commissioning, monitoring and maintenance of field devices is proving to be visionary (Schmitt, 2013). The combination of modern interaction paradigms and modular software components allows an increase in flexibility and dynamism in the function deployment and extension for industrial field devices (e.g. reloading of field device apps), as well as significantly higher usability and effectiveness in their application (e.g. automatic software update mechanism). Additionally, the use of standardized communication and user interfaces enable wireless, mobile interaction with field devices.

Since the introduction of the iPhone in 2007, so called app-concepts have been established in the field of consumer electronics to simplify the installation, upgrade and uninstalling of apps on mobile devices. In most cases, a so called "App Store" in the network serves as a digital distribution platform for the modular software components. This service allows users to search and download apps automatically from a catalog. The main advantage of this concept is the efficient management and maintenance of the apps across the entire software life cycle. In addition, the apps are provided to the user as modular software components via standardized software interfaces. Another advantage is the openness of the platforms, allowing so called third-party applications, which means that a decoupling of software and hardware manufacturing is becoming possible.

Although there are already a few companies offering industrial apps, these software-components are mostly marketing-oriented (e.g. portfolio catalog). In addition, there are already some vendor-specific approaches, e.g. which provide apps on mobile devices, in order to address their own field devices via fieldbus and PLC. These are usually monitoring functionalities (Bosch, 2013; Siemens 2013). However, concerning the latest research literature, clearly there are no holistic approaches for the transfer of established app-concepts out of the field of consumer electronics and into the industrial application, to enable a dynamic and flexible adaption of field device functionality by using apps on field devices.

Nowadays complex field devices are usually developed and sold as a complete, self-contained unit. For the customer the firmware is normally a fixed and unchangeable part of the product. In the prospect of flexibility and changeability demands, this approach is not very promising. Due to this, new interaction concepts for industrial field device are needed, e.g. to provide a separation between the sale of hardware, including a firmware framework serving as a software platform, and the sale of available software components in the form of apps as a functional extension (e.g. monitoring app). The apps can either be developed and offered by the component manufacturer or from the so-called third-parties. Conceivable are both fully programmable field devices, as well as the enabling of functionality by the user downloading apps on the field device.

\section{Objective and general approach of the industrial App-Framework}

The major objective is therefore to develop and implement a multi-vendor app-framework that allows dynamic feature deployment and extension by using apps on industrial field devices complemented by a user-friendly and effective field device interaction. To achieve this superior objective, three sub-objectives are defined, which are described in the section below.

First, a reference architecture is developed and implemented as a basis for the implementation of the proposed app-framework. The developed reference architecture provides an abstract, ideal-typical description of the system, taking into account all system components and their inter-relationships. Thus, a high degree of reusability and scalability for the integration of the app-framework into factory systems is ensured. By the following instantiation of the reference architecture, an implementation design results, which specifies the defined system components based on specific hardware and software technologies. Thus, the first sub-objective provides the implementation basis for the realization of the app-framework.

In the next step, the concrete implementation of the instantiated reference architecture and thus, the exemplary, technological implementation of the app-framework for field devices takes place. The requirements are provided by the implementation design. According to the specification, the hardware, including the software programs, are developed and designed. As a result, the exemplary realization of the previously defined system components in the form of specific software elements evolves. Each software element can be accurately assigned to a system component of the reference architecture. The 
individual system components of the reference architecture put together result into an overall system and form the basis for the last sub-objective.

In a last step, the development and implementation of a comprehensible technology-demonstrator takes place. Therefore the previously implemented software components of the app-Framework are integrated into a coherent overall system. Based on an industry-relevant use case, the developed and implemented app-framework for industrial field devices can be demonstrated and evaluated. The technology-demonstrator serves primarily as a technical proof of concept of the app-framework.

\section{Description of the industrial App-Framework}

In order to realize a dynamic feature deployment and extension by using apps on industrial field devices, the developed reference architecture provides the system components, which fulfill crucial functions. The system components are: the industrial App Store, device registry, mobile user interface, field device middleware, and cloud-based runtime environment.

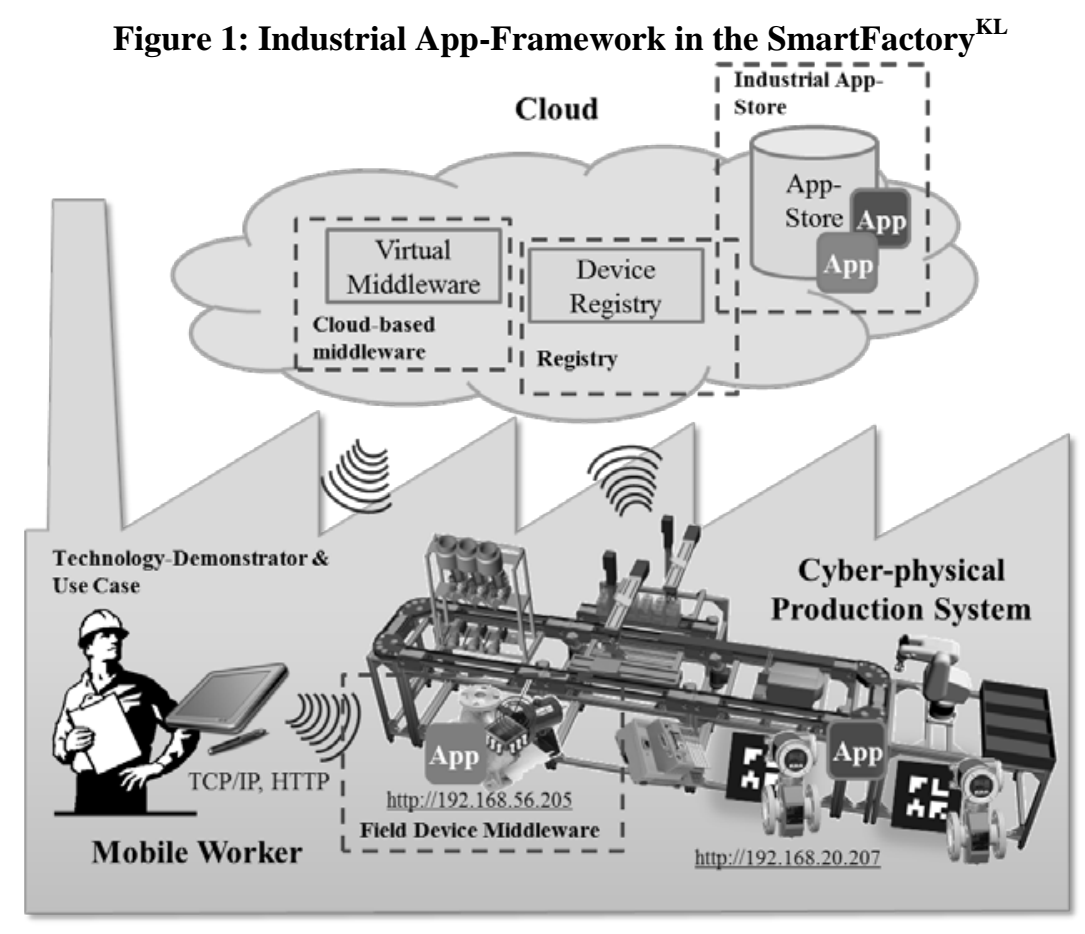

Source: German Research Center for Artificial Intelligence

\subsection{Apps for Field Devices and industrial App Store}

The app-framework provides manufacturer-independent modular software components in the form of field device apps, which are provided for the user in the factory network. In this so-called industrial App Store, there is a vendor catalog, which lists all available apps for registered field devices. The registration of available field devices is done via a factory-internal device registry, which runs in an internal cloud. For this purpose all available field devices in the environment subscribe dynamically to this registry (e.g. via an electronic device description). Via standardized, mobile interaction devices (e.g. Tablet) the user is able to access the registry. Therefore, on the one hand, the registry provides an interface to the user and, on the other hand, a standardized interface to the field device. Alternatively, field devices can be equipped with passive data memories. Via the so-called "touch and connect method" (Floerchinger, 2011), the data memories can be read out to initiate communication between the mobile and the field device. The apps can be installed and run directly on the field device. Thus, not only a flexible function deployment and extension is possible (e.g. dynamic reloading of apps), but also a comfortable management of software components across the entire software life cycle (installation, update, uninstall). The fact that apps are not running on the mobile device anymore, but directly on the field device, is a visionary aspect. 


\subsection{Mobile User Interfaces}

Standardized, mobile user interfaces are used for interaction with the field devices to support the user efficiently during configuration or reconfiguration of the field devices on-site in the factory (e.g. during maintenance and repair work). A key innovation is the removal of the user interface from the field device. By separating the user interface and the app itself device-stationary user interfaces can be overcome (e.g. display and buttons on the device) and due to this, significantly smaller and less expensive field devices can be realized because the user interface isn't integrated anymore. From a technological and economic perspective, this approach seems future-orientated concerning the interaction with intelligent field devices.

\subsection{Middleware for field devices}

A field device middleware provides a uniform runtime environment for the device specific apps. This is the technological enabler to update or adapt field device functionalities via reloading apps on the device. An essential component of the middleware is the so called life cycle manager (LCM), which provides an interface to the user's mobile device and to the registry. The LCM has the task to administrate (install, update, uninstall) the apps on the field device. In addition, the middleware provides drivers to enable interaction with the sensors and actors. Thus, proprietary software solutions, which are usually required for all field devices, are replaced by uniform apps. As a result, third-party suppliers are also able to develop and distribute apps independently of the manufacturing of hardware.

\subsection{Cloud Application}

Since the app-framework for field devices can be integrated easily into existing production lines, a sustainable approach is given to the users and developers of field devices. For current field device generations that do not have sufficient processing-intelligence, a cloud-based execution environment is provided for Apps. This approach provides a virtualized version of the middleware developed for field devices. The processing intelligence is therefore not an absolute requirement in the field device (e.g. simple inductive sensor). The management and the interaction with the apps in the virtualized runtime environment is analogous to the interaction with the apps on the field devices. Another purpose of the Cloud is to provide a general, factory-internal runtime environment for the industrial App Store and the registry.

\section{Conclusions and Outlook}

Changeable and flexible factory systems equally imply the need for a consequent adaption of the field device functionalities according to the changing production conditions. Especially in the area of field device integration (e.g. during commissioning or maintenance) there are primarily stationary and proprietary software solutions and user interfaces available.

Thereby, the user is more frequently faced with an increasing variety of field devices from different manufacturers, which leads not just to a considerable effort for configuration or reconfiguration of field devices, but also carries the risk of incorrect operation by the user. The introduction of multivendor app-framework allows dynamic and flexible feature deployment and expansion by using apps on field devices. Furthermore a much more user-friendly and effective interaction with field devices can be realized. As a result, proprietary software solutions and device-stationary user interfaces can be overcome and replaced by uniform, cross-vendor solutions.

Important aspects of the presented app-framework are: How to transfer established interaction technologies and metaphors from the field of consumer electronics into industrial applications and which are the essential constraints? How to develop a reference architecture for the app-framework and which are the central software components of the reference architecture? Which are the constraints and how to implement the interfaces of the field device middleware to realize apps on field devices? How can a holistic approach be established, which also addresses field devices that do not have an embedded processing intelligence (e.g. via cloud-based approach)? And last but not least, how to separate the graphical user interface from the app itself.

The aim of the paper is to give a preliminary insight regarding the current work. In addition to an extensive requirements analysis, so far, a prototypical software architecture has been developed. Beyond this, the implementation of selected software components has already started. Thus, a prototypical device registry and an industrial App Store have been implemented in the SmartFactory ${ }^{\mathrm{KL}}$ (Zuehlke, 2009; Zuehlke, 2010), our real-world test bed operated by the department for Innovative Factory Systems at the 
German Research Center for Artificial Intelligence. In a next step the middleware for field devices and a cloud-based runtime environment (e.g. for normal sensors and actors) have to be developed. In a final step, a demonstrator, which integrates the developed software components in a coherent overall-system, has to be developed as a proof of concept and for evaluating the developed app-framework for industrial field devices. The app-framework for dynamic function deployment and extension will be implemented and demonstrated in the SmartFactory ${ }^{\mathrm{KL}}$.

\section{References}

- Abele, E.; Reinhart, G. (2011) Zukunft der Produktion: Herausforderungen, Forschungsfelder, Chancen. Carl Hanser Verlag, München.

- Bosch on mobile devices (2013) Apps from Bosch. Available at http://apps.bosch.com/en/index.htm, 18.10.2013.

- Broy, M., Kargermann, H., Achatz, R. (2010) Agenda Cyber Physical Systems, Outlines of a new Research Domain, acatech, Berlin, Germany.

- EDDL (2013) Electronic Device Description Language. Available at http://www.eddl.org/Pages/default.aspx, 17.10.2013.

- FDT Group (2013) Field Device Tool / Device Type Manager. Available at http://www.fdtgroup.org/, 17.10.2013.

- Floerchinger, F.; Schmitt, M. (2011) A Concept for a user-friendly first Communication Initiation between Stationary Field Devices and Mobile Interaction Devices. In S. Bittanti, A. Cenedese, S. Zampieri (ed.), Proc. of the 18th International Federation of Automatic Control (IFAC) World Congress, 1614-1619, International Federation of Automatic Control, Milano, Italy.

- Lee, A. E., (2008), Cyber Physical Systems: Design Challenges, Int. Symposium Object / Component / Service-Oriented Real-Time Distributed Computing (ISORC), pp. 363-369, Orlando, SUA.

- Schmitt, M.; Meixner, G.; Gorecky, D.; Seissler, M.; Loskyll, M. (2013) Mobile Interaction Technologies in the Factory of the Future. IFAC/IFIP/IFORS/IEA Symposium on Analysis, Design, and Evaluation of Human-Machine Systems. August 11-15, Las Vegas, USA.

- Siemens (2013) Siemens Apps. Available at http://www.siemens.de/mobile/apps/Pages/Home.aspx, 18.10.2013.

- Zuehlke, D. (2009) SmartFactory - A Vision becomes Reality. In: Keynote Papers of the 13th IFAC Symposium on Information Control Problems in Manufacturing (INCOM 09), June 3-5, Moscow, Russian Federation, ICS / RAS.

- Zuehlke, D. (2010) SmartFactory - Towards a Factory-of-Things. Annual Reviews in Control, volume 34, 129-138. 